\title{
Dècade: solo dieci giorni o anche dieci anni?
}

\section{Giuseppe Patota}

PUBBLICATO: 05 MAGGIO 2020

\section{Quesito:}

Alcuni lettori ci chiedono se l'uso recente di decade per indicare un 'periodo di dieci anni' sia da imputarsi all'influenza della forma inglese decade oppure sia una possibilità presente anche nella nostra lingua.

\section{Décade: solo dieci giorni o anche dieci anni?}

D écade è una parola di tradizione dotta, che l'italiano ha assunto dal latino scritto décadem, a sua volta proveniente dal greco dekás, dekádos, da déka 'dieci'. Dal significato più ampio del termine, che fu 'decina', 'complesso di dieci cose o persone', sono derivati e continuano a derivarne altri: in passato la parola decade ha indicato la 'paga dei soldati e degli attori', che tradizionalmente venivano retribuiti ogni dieci giorni; nel presente il termine ha assunto, in contesti particolari (per esempio l'elettrotecnica, la telefonia o l'informatica) una valenza tecnica, indicando vari tipi di decine (di transistori, di resistori, ecc.) e anche, più in generale, un intervallo di valori assunti da una grandezza fisica, spec. una frequenza o una lunghezza d'onda, i cui estremi stanno tra loro nel rapporto di i a Io' (Zingarelli 2019). Ma il significato di gran lunga più ricorrente di decade, documentato in tutti i vocabolari che descrivono l'italiano contemporaneo, è quello di 'periodo di dieci giorni' e, in particolare, quello di 'ciascuno dei tre periodi di circa dieci giorni nel quali si divide un mese' (Garzanti 2013): "Gli esami si svolgeranno nella prima decade di giugno»; «Il lavoro andrà consegnato entro la terza decade di luglio".

Gli utenti che ci segnalano, talvolta sorpresi talaltra allarmati, la diffusione, nellitaliano attuale, di un nuovo significato di decade, quello di 'decennio', 'periodo di dieci anni', pensano a un probabile influsso dell'inglese, in cui decade ha, per l'appunto, questo valore. Due nostri vocabolari - il GRADIT e il Vocabolario Treccani 2008 - documentano quest'accezione, qualificandola come rara (o non comune, il che è lo stesso).

Che se ne trovino esempi nell'uso attuale è fuori discussione: bastino, a dimostrarlo, i tre che produco qui sotto, ricavati da una rapidissima ricerca in rete:

Le prime decadi del Novecento videro affermarsi un neokantismo discreto, fino a quando verso la metà degli anni trenta ebbero inizio movimenti importanti per la cultura filosofica della città. (Accademia delle Scienze di Torino, La filosofia a Torino, dossier consultabile in rete);

Proprio il XX Secolo, infatti, rappresenta il fil rouge del nuovo ordinamento espositivo: raccontato, in sintesi (da cui il titolo della mostra), attraverso le sue decadi, il nuovo allestimento consentirà di intraprendere un viaggio nella memoria, attraverso oggetti, stili del design e la storia dei materiali polimerici. ("Il Mattino", I3/II/2019);

Ci sono Mario Draghi e Sergio Marchionne tra le personalità che hanno lasciato la loro impronta sul decennio in via di chiusura con la fine del 2019. Una decade, la seconda del nuovo millennio, iniziata con il mantra dell'austerità per rimettere in sesto i bilanci picconati dalla Grande recessione. ("la Repubblica", 27/12/2019). 
È possibile che l'attuale diffusione di questa particolare decade sia dovuta a un influsso dell'inglese. Ma l'uso del termine nel senso di 'decennio' è italianissimo: il lettore ne avrà la prova consultando nel sito dell'Accademia della Crusca il GDLI, che attesta l'uso di decade nel significato di decennio in Luigi Lanzi (I732-I8Io), in Giosue Carducci (I835-1907) e in Emilio Cecchi (I884-I966).

\section{Cita come:}

Giuseppe Patota, Dècade: solo dieci giorni o anche dieci anni? , "Italiano digitale", 2020, XIII, 2020/2 (aprile-giugno)

DOI: $10.35948 / 2532-9006 / 2020.3325$

Copyright 2020 Accademia della Crusca

Pubblicato con licenza creative commons CC BY-NC-ND 